\title{
On Elastin in Aorta of SHRSP (2)
}

\author{
Hiroyuki Iто, Kazuo Yамамото, and Kozo Окамото
}

It was shown in our previous study that the aging process in the aortic elastin of SHRSP proceeded earlier than in Wistar-Kyoto rats (Jap Heart J 18:592, 1977). In order to clarify the character of the arterial changes in SHRSP, aortic elastin was isolated and its amino acid composition was examined chronologically, in comparison with that of Wistar-Kyoto rats.

\section{Materials and Methods:}

Male SHRSP and Wistar-Kyoto rats as control were mainly used for this study. Some of the SHRSP were fed with a $50 \%$ white fish meal or $50 \%$ casein diet by weight from 35 days after birth.

After sacrificing these rats at various ages, the thoracic aorta was isolated from each animal and adhering tissues were separated. Five of 6 rats were used for each experiment. These aortas were homogenized in a $0.25 \mathrm{M}$ sucrose solution using Virtis homogenizer. Nuclear fraction was obtained by centrifuging at $1,000 \times \mathrm{g}$ for $10 \mathrm{~min}$. According to Partridge's method (Biochem J $61: 11,1955$ ), water-insoluble elastin was prepared from this fraction (Partridge elastin).

Some of these materials were treated with a $0.1 \mathrm{~N} \mathrm{NaOH}$ at $98^{\circ} \mathrm{C}$ for $45 \mathrm{~min}$ to prepare an alkali-insoluble elastin (Lansing elastin) according to Lansing's method (Anat Record 114:555, 1952). The alkali-soluble fraction was discarded.

The insoluble elastins were weighed and its proportion in the aorta was examined. Five mg of elastin was hydrolized in $6 \mathrm{~N} \mathrm{HCI}$ at $110^{\circ} \mathrm{C}$ for 72 hours and amino acid analysis was performed using a JOEL amino acid analyser.

\section{Results:}

1) Proportion of elastin in aorta

As previously reported, the proportion of Partridge elastin was high in young animals, decreased with age, and became nearly constant after 4 months of age. There were certain differences in the proportion of elastin between SHRSP and control rats at similar ages, a higher proportion in the former than in the latter. Furthermore, in the SHRSP fed with white fish meal or casein diet, the proportion of elastin in aorta was higher than in the SHRSP fed with a stock chow diet. This became more remarkable with age.

On the other hand, in comparison with Partridge elastin, the proportion of Lansing elastin in the aorta was lower in both strains. This proportion decreased with age. After 4 months of age, the proportion of this alkali-insoluble elastin in SHRSP became lower than in Wistar-Kyoto rats.

2) Amino acid composition of elastin

As previously reported, the proportion of polar amino acids was low in young animals but increased with age. There were certain differences between SHRSP and Wistar-Kyoto rats, a higher proportion in the former than in the latter after 3

\footnotetext{
From the Department of Pathology, Kinki University School of Medicine, Osaka-fu.
} 
months of age. The content of desmosine and isodesmosine in Partridge elastins were nearly constant in Wistar-Kyoto rats. But in SHRSP, these amino acids decreased slightly with age, being 2.4 at 1 month of age and 1.8 at 8 months of age (residues per 1,000 residues). On the other hand, while the content of lysine in SHRSP increased with age, no chronological changes were found in Wistar-Kyoto rats.

After the extraction of a alkali-soluble fraction with $\mathrm{NaOH}$, there were no significant differences in the proportion of polar amino acids of alkali-insoluble elastins not only in both strains but also in young and old animals : all Lansing elastins showed similar amino acid compositions.

\section{Discussion:}

In this experiment, the proportion of alkali-insoluble elastin by the Lansing method markedly decreased with age and became lower in SHRSP than in WistarKyoto rats after 4 months of age. Moreover, no significant differences were found in amino acid compositions of all the alkali-insoluble elastin preparations. These results indicate that the advanced proceeding of the aging process in aortic elastin of SHRSP is due to the increase of alkali-soluble fraction in Partridge elastin and the decrease of alkali-insoluble elastin. This increase of alkali-soluble fraction may be due to the deposition of degradation products of Lansing elastin.

On the other hand, the tendency for a decrease in the content of desmosine and isodesmosine and the increase in the content of lysine indicates the decrease of biosynthesis of elastin in aorta of SHRSP of an advanced age, probably resulting in a decrease of Lansing elastin. These results suggest a decrease of elasticity in the aorta of SHRSP.

An increase of the proportion of Partridge elastin was found in the aorta of SHRSP fed with white fish meal and casein diet, but no significant differences in amino acid composition was found in comparison with SHRSP fed with stock chow diet. This result seems to indicate the possibility that the stimulation of elastin biosynthesis may occur in aorta of SHRSP fed with these experimental diets.

This study was supported by the Science and Technology Agency of Japanese Government, Ministry of Education, the Japanese Society for the Promotion of Science, and the National Heart and Lung Institute of NIH (USA) (HL17754-03). 